\title{
Stabilization of photon-number states via single-photon corrections: a first convergence analysis under an ideal set-up
}

\author{
H. B. Silveira \\ P. S. Pereira da Silva \\ P. Rouchon
}

\begin{abstract}
This paper presents a first mathematical convergence analysis of a Fock states feedback stabilization scheme via single-photon corrections. This measurement-based feedback has been developed and experimentally tested in 2012 by the cavity quantum electrodynamics group of Serge Haroche and Jean-Michel Raimond. Here, we consider the infinitedimensional Markov model corresponding to the ideal setup where detection errors and feedback delays have been disregarded. In this ideal context, we show that any goal Fock state can be stabilized by a Lyapunov-based feedback for any initial quantum state belonging to the dense subset of finite rank density operators with support in a finite photon-number sub-space. Closed-loop simulations illustrate the performance of the feedback law.
\end{abstract}

\section{INTRODUCTION}

In [8], a photon-number states (Fock state) feedback stabilization scheme via single-photon corrections was described and experimentally tested. Such control problem is relevant for quantum information applications [6], [4]. The quantum state $\rho$ corresponds to the density operator of a microwave field stored inside a super-conducting cavity and described as a quantum harmonic oscillator. At each sample step $k \in \mathbb{N}$, a probe atom is launched inside the cavity. The measurement outcome $y_{k}$ detected by a sensor is the energy-state of this probe atom after its interaction with the microwave field. Each probe atom is considered as a two-level system: either it is detected in the lowest energy state $|g\rangle$, or the highest energy state $|e\rangle$. Consequently, the measurement outcomes corresponds to a discrete-valued output $y_{k}$ with only two distinct possibilities: $g$ or $e$. Similarly, the control inputs $u_{k}$ are also discrete-valued with 3 distinct possibilities: $-1,0,+1$. The open-loop value $u_{k}=0$ corresponds to a dispersive atom/field interaction: it achieves in fact a Quantum Non-Demolition measurement of Fock states [2]. The two other values $u_{k}= \pm 1$ correspond to resonant atom/field interactions where the probe atom and the field exchange energy quanta: these values achieve single-photon corrections.

H. B. Silveira was fully supported by CNPq (National Counsel for the Scientific and Technological Development), Ministry of Science and Technology, Brazil, as a visiting professor at Centre Automatique et Systèmes, Mines ParisTech. P. S. Pereira da Silva was partially supported by CNPq. P. Rouchon was partially supported by Projet Blanc ANR-2011-BS01-017-01 EMAQS.

H. B. Silveira is with Departamento de Automação e Sistemas (DAS), Federal University of Santa Catarina (UFSC), Florianópolis, Brazil hector.silveira@ufsc.br

P. S. Pereira da Silva is with Escola Politécnica - PTC, University of São Paulo (USP), São Paulo, Brazil paulo@lac.usp.br

P. Rouchon is with Centre Automatique et Systèmes, Mines ParisTech, PSL Research University, Paris, France pierre.rouchon@mines-paristech.fr
Although the feedback law proposed and implemented in [8] considered imperfect detections on $y_{k}$ and delays in the control, here we focus on an ideal-set up, that is, detection errors and control delays have been disregarded. Theorem 2 shows that, by adding an arbitrarily small term to the Lyapunov function used in [8], one ensures almost sure global stabilization of any goal Fock state for the closedloop ideal set-up. This is achieved by relying on an infinitedimensional Markov model of the ideal set-up that takes into account the back-action of the measurement outcome $y_{k}$ on the quantum state $\rho_{k+1}$.

Loosely speaking, in [8], the control value $u_{k}$ at each sampling step $k$ was chosen so as to minimize the conditional expectation of the Lyapunov function $V\left(\rho_{k}\right)=$ $\operatorname{Tr}\left(d(\boldsymbol{N}) \rho_{k}\right)$, where $\boldsymbol{N}$ is the photon-number operator, $d(n)=(n-\bar{n})^{2}$ and $\bar{\rho}=|\bar{n}\rangle\langle\bar{n}|$ is the goal Fock state. However, in closed-loop, the difference between such $V$ and its conditional expectation is not strictly positive: such $V$ does not become a strict Lyapunov function in closedloop and additional arguments have to be considered to prove convergence. These additional arguments are related to Lasalle invariance. They are well established in a smooth context where the control $u$ is a smooth function of the state $\rho$. This cannot be the case here since $u$ is a discrete-valued control. In order to overcome such technical difficulties, we propose, similarly to [1], to add the arbitrarily small term $-\epsilon \sum_{n=0}^{\infty}\left(\left\langle n\left|\rho_{k}\right| n\right\rangle\right)^{2}$ to $V\left(\rho_{k}\right)$, where $\epsilon>0$. This slightly modified control-Lyapunov function becomes then a strictLyapunov function in closed-loop that simplifies notably the convergence analysis. Moreover, the developed convergence analysis is done in the infinite-dimensional setting in the following sense: we show that, for any initial density operator $\rho_{0}$ with a finite photon-number support $\left(\rho_{0}|n\rangle=0\right.$ for $n$ large enough), the closed-loop trajectory $k \mapsto \rho_{k}$ remains also with a finite photon-number support with a uniform bound on the maximum photon-number. This almost finitedimensional behavior simplifies the convergence analysis despite the fact that such condition on $\rho_{0}$ is met on a dense subset of density operators (Hilbert-Schmidt topology on the Banach space of Hilbert-Schmidt self-adjoint operators).

The paper is organized as follows. Section III presents the ideal Markov model of the experimental set-up of the controlled microwave super-conducting cavity reported in [8] and precisely formulates the Fock state stabilization problem here treated (see Definition [1). Section III establishes the proposed solution to the control problem in two distinct parts. Firstly, Section III-A considers the case where the initial condition $\rho_{0}$ is a diagonal density operator (see Theorem 1 ). 
Only the main ideas of the convergence proof are outlined. The technical details are given in Section $\mathrm{V}$, Afterwards, in Section III-B, the main result of the paper is presented: the general solution is obtained from Theorem 1 for $\rho_{0}$ belonging to a dense subset (see Theorem 2). The simulation results are exhibited in Section IV The proof of some intermediate results and computations required in Sections III] and V are presented in Appendices $\mathrm{B}, \mathrm{G}$. Finally, the concluding remarks are given in Section VI.

\section{IDEAL MARKOV MODEL}

Denote by $\mathcal{H}$ the separable complex Hilbert space $L_{2}(\mathbb{C})$ with orthonormal basis $\{|n\rangle, n \in \mathbb{N}\}$ of Fock states (photonnumber). Hence, $\mathcal{H}=\left\{\sum_{n \in \mathbb{N}} \psi_{n}|n\rangle,\left(\psi_{0}, \psi_{1}, \ldots\right) \in\right.$ $\left.l_{2}(\mathbb{C})\right\}$. Let $\mathbb{D}$ be the set of all density operators on $\mathcal{H}$, that is, the set of trace-class, self-adjoint, non-negative operators on $\mathcal{H}$ with unit trace. The sample step, corresponding to a sampling period around $100 \mu \mathrm{s}$, is indexed by $k \in \mathbb{N}=$ $\{0,1,2, \ldots\}, u_{k} \in\{-1,0,1\}$ is the control, $\rho_{k} \in \mathbb{D}$ the quantum state and $y_{k} \in\{g, e\}$ the measurement outcome. The ideal Markov model of the controlled microwave superconducting cavity used in [8] is given by:

$$
\rho_{k+1}=\left\{\begin{array}{l}
\rho_{k+1}^{g}=\frac{\boldsymbol{M}_{g}\left(u_{k}\right) \rho_{k} \boldsymbol{M}_{g}^{\dagger}\left(u_{k}\right)}{\operatorname{Tr}\left(\boldsymbol{M}_{g}\left(u_{k}\right) \rho_{k} \boldsymbol{M}_{g}^{\dagger}\left(u_{k}\right)\right)} \text { when } y_{k}=g, \\
\rho_{k+1}^{e}=\frac{\boldsymbol{M}_{e}\left(u_{k}\right) \rho_{k} \boldsymbol{M}_{e}^{\dagger}\left(u_{k}\right)}{\operatorname{Tr}\left(\boldsymbol{M}_{e}\left(u_{k}\right) \rho_{k} \boldsymbol{M}_{e}^{\dagger}\left(u_{k}\right)\right)} \text { when } y_{k}=e,
\end{array}\right.
$$

where the measurements outcomes $y_{k}=g$ and $y_{k}=e$ occur with probabilities $11 p_{g, k}=\operatorname{Tr}\left(\boldsymbol{M}_{g}\left(u_{k}\right) \rho_{k} \boldsymbol{M}_{g}^{\dagger}\left(u_{k}\right)\right)$ and $p_{e, k}=\operatorname{Tr}\left(\boldsymbol{M}_{e}\left(u_{k}\right) \rho_{k} \boldsymbol{M}_{e}^{\dagger}\left(u_{k}\right)\right)=1-p_{g, k}$, respectively, $u_{k}=0$ corresponds to a dispersive interaction of the launched atom with the cavity field (Quantum NonDemolition measurement of photons)

$$
\boldsymbol{M}_{g}(0)=\cos \left(\frac{\phi_{0} \boldsymbol{N}+\phi_{R}}{2}\right), \boldsymbol{M}_{e}(0)=\sin \left(\frac{\phi_{0} \boldsymbol{N}+\phi_{R}}{2}\right),
$$

when $u_{k}=+1$ the atom enters the cavity in the state $|e\rangle$ with a resonant interaction with the cavity field

$$
\boldsymbol{M}_{g}(+1)=\frac{\sin \left(\frac{\theta_{0}}{2} \sqrt{\boldsymbol{N}}\right)}{\sqrt{\boldsymbol{N}}} \boldsymbol{a}^{\dagger}, \boldsymbol{M}_{e}(+1)=\cos \left(\frac{\theta_{0}}{2} \sqrt{\boldsymbol{N}+1}\right),
$$

when $u_{k}=-1$ it enters in $|g\rangle$ with a resonant interaction

$$
M_{g}(-1)=\cos \left(\frac{\theta_{0}}{2} \sqrt{\boldsymbol{N}}\right), \boldsymbol{M}_{e}(-1)=\boldsymbol{a} \frac{\sin \left(\frac{\theta_{0}}{2} \sqrt{\boldsymbol{N}}\right)}{\sqrt{\boldsymbol{N}}},
$$

and $\phi_{0}, \phi_{R}, \theta_{0} \in \mathbb{R}$ are adjustable control parameters. For each $u \in\{-1,0,1\}, \boldsymbol{M}_{g}(u)$ and $\boldsymbol{M}_{e}(u)$ are (linear) operators on $\mathcal{H}$ defined in the obvious way according to the definitions in Appendix $\mathrm{A}$ They are indeed well-defined operators on $\mathcal{H}$, despite the fact that $\boldsymbol{a}$ and $\boldsymbol{a}^{\dagger}$ are unbounded

\footnotetext{
${ }^{1}$ As usual in quantum physics, it is here assumed that the measurement outcome $y_{k}=y$ cannot occur when $\operatorname{Tr}\left(\boldsymbol{M}_{y}\left(u_{k}\right) \rho_{k} \boldsymbol{M}_{y}^{\dagger}\left(u_{k}\right)\right)=0$, for $y=g, e$.

${ }^{2}$ For instance, $\boldsymbol{M}_{g}(+1)|n\rangle=\left(\sin \left(\frac{\theta_{0}}{2} \sqrt{\boldsymbol{N}}\right) / \sqrt{\boldsymbol{N}}\right) \sqrt{n+1}|n+1\rangle=$ $\sin \left(\frac{\theta_{0}}{2} \sqrt{n+1}\right)|n+1\rangle$. In order for the definition of $\boldsymbol{M}_{e}(-1)$ to be consistent, it is assumed $\sin (0) / 0=1$.
}

operators. It is clear that $\boldsymbol{M}_{g}(u), \boldsymbol{M}_{e}(u)$ are bounded operators on $\mathcal{H}$ with $\boldsymbol{M}_{g}^{\dagger}(u) \boldsymbol{M}_{g}(u)+\boldsymbol{M}_{e}^{\dagger}(u) \boldsymbol{M}_{e}(u)=\boldsymbol{I}$ (identity operator), $\boldsymbol{M}_{e}(-1)=\boldsymbol{M}_{g}^{\dagger}(+1)=\boldsymbol{a} \sin \left(\frac{\theta_{0}}{2} \sqrt{\boldsymbol{N}}\right) / \sqrt{\boldsymbol{N}}$, and $\boldsymbol{M}_{g}(-1), \boldsymbol{M}_{g}(0), \boldsymbol{M}_{e}(0), \boldsymbol{M}_{e}(+1)$ are self-adjoint. It is easy to see that if the initial condition $\rho_{0}$ is a density operator then, for all realizations of the ideal Markov process (1)-(4), $\rho_{k}$ is a density operator for $k \in \mathbb{N}$.

Notice that $\bar{\rho}=|\bar{n}\rangle\langle\bar{n}|$ is a steady state of the Markov process (1)-(4) with $u_{k}=0$, where $\bar{n} \in \mathbb{N}$ is arbitrary. The control problem here treated is given as follows:

Definition 1: For the ideal Markov process (1)-(4), the control problem is to find a feedback law $u_{k}=f\left(\rho_{k}\right)$ such that, given an initial condition $\rho_{0}$ and $\bar{n} \in \mathbb{N}$, the closed-loop trajectory $\rho_{k}$ converges almost surely towards the goal Fock state $\bar{\rho}=|\bar{n}\rangle\langle\bar{n}|$ as $k \rightarrow \infty$.

The almost sure convergence above is with respect to the probabilities amplitudes $P_{n}(\rho)=\operatorname{Tr}(|n\rangle\langle n| \rho)=\langle n|\rho| n\rangle$ of $\rho$, that is, $\lim _{k \rightarrow \infty} P_{n}\left(\rho_{k}\right)=P_{n}(\bar{\rho})$ for each $n \in \mathbb{N}$. In other words, $\lim _{k \rightarrow \infty} P_{\bar{n}}\left(\rho_{k}\right)=1$ and $\lim _{k \rightarrow \infty} P_{n}\left(\rho_{k}\right)=0$ when $n \neq \bar{n}$. The solution proposed in this paper for the control problem above is developed in the next section.

\section{STABILIZATION OF FOCK STATES}

Given any operator $A: \mathcal{H} \rightarrow \mathcal{H}$, let $A_{m n}=\langle m|A| n\rangle$ for $m, n \in \mathbb{N}$. Hence, $A_{n n}$ is the $n$-th diagonal element of $A$, while $A_{m n}$ with $m \neq n$ correspond to its "off-diagonal" elements. One says that the operator $A$ is diagonal when $A_{m n}=0$ for all $m, n \in \mathbb{N}$ with $m \neq n$. One shall begin by solving the control problem given in Definition 1 in the particular case where the initial condition $\rho_{0}$ is diagonal (see Theorem 11 in Section [II-A). Afterwards, in Section [II-B, the solution to the general non-commutative case is presented (see Theorem 2): its solution relies essentially on the diagonal case.

\section{A. Diagonal case}

For each $n^{*} \in \mathbb{N}$, define $3^{3}$

$$
D_{n^{*}}=\left\{\rho \in \mathbb{D} \mid \rho \text { is diagonal and } \rho|n\rangle=0, \forall n>n^{*}\right\} .
$$

Consider the set $D_{*}=\bigcup_{n^{*} \in \mathbb{N}} D_{n^{*}} \subset \mathbb{D}$. Note that $D_{n^{*}} \subset$ $D_{n^{*}+1}$, and that each element $\rho$ of $D_{*}$ is "finite dimensional" in the following sense: $\rho \in \mathbb{D}$ is in $D_{n^{*}}$ if and only if $\rho=\sum_{n=0}^{n^{*}} \rho_{n n}|n\rangle\langle n|$, and $\rho \in D_{n^{*}}$ may be considered as an operator from $\mathcal{H}$ to the finite-dimensional space $\mathcal{H}_{n^{*}}=$ $\operatorname{span}\left\{|0\rangle, \ldots,\left|n^{*}\right\rangle\right\}$, or as a density matrix on $\mathcal{H}_{n^{*}}$. One defines the functions $n_{\min }: D_{*} \rightarrow \mathbb{N}, n_{\max }: D_{*} \rightarrow \mathbb{N}$ and $n_{\text {length }}: D_{*} \rightarrow \mathbb{N}$ respectively by:

- $n_{\min }(\rho)$ is the smallest $n \in \mathbb{N}$ such that $\rho|n\rangle \neq 0$;

- $n_{\max }(\rho)$ is the greatest $n \in \mathbb{N}$ such that $\rho|n\rangle \neq 0$;

- $n_{\text {length }}(\rho)=n_{\max }(\rho)-n_{\min }(\rho)$.

It is clear that, given $\rho \in D_{*}$, one has $\rho \in D_{n^{*}}$ if and only if $n_{\max }(\rho) \leq n^{*}$. The next result exhibits the properties of the state $\rho_{k}$ of (1)-(4) with respect to these functions.

\footnotetext{
${ }^{3}$ Note that if $\rho=|n\rangle\langle n|$ for some $n \in \mathbb{N}$, then $\rho \in D_{n}$.
} 
Proposition 1: For every realization of the ideal Markov process (1)-(4) with initial condition $\rho_{0} \in D_{*}$, one has that $\rho_{k} \in D_{*}$ for all $k \in \mathbb{N}$ with:

- If $u_{k}=0$ or $u_{k}=-1$, then $n_{\max }\left(\rho_{k+1}\right) \leq n_{\max }\left(\rho_{k}\right)$ and $n_{\text {length }}\left(\rho_{k+1}\right) \leq n_{\text {length }}\left(\rho_{k}\right)$;

- If $u_{k}=+1$, then $n_{\max }\left(\rho_{k+1}\right) \leq n_{\max }\left(\rho_{k}\right)+1$ and $n_{\text {length }}\left(\rho_{k+1}\right) \leq n_{\text {length }}\left(\rho_{k}\right)$.

Proof: See Appendix B

Take a goal photon-number $\bar{n} \in \mathbb{N}$. As in [1], consider the following Lyapunov function $V_{\epsilon}: D_{*} \rightarrow \mathbb{R}$ defined as

$$
V_{\epsilon}(\rho)=\operatorname{Tr}(d(\boldsymbol{N}) \rho)-\epsilon \sum_{n \in \mathbb{N}} \rho_{n n}^{2}, \quad \text { for } \rho \in D_{*},
$$

where $\epsilon>0$ is a real number and $d(n)=(n-\bar{n})^{2}$ as defined in [8]. The feedback law $u: D_{*} \rightarrow\{-1,0,1\}$ is given by

$$
u=f(\rho) \triangleq \underset{v \in\{-1,0,1\}}{\operatorname{Argmin}} \mathbb{E}\left[V_{\epsilon}\left(\rho_{k+1}\right) \mid \rho_{k}=\rho, u_{k}=v\right] .
$$

Note that for each $\rho \in D_{*}$ and $n^{*} \geq n_{\max }(\rho), d(\boldsymbol{N}) \rho$ in (5) is a well-defined self-adjoint, non-negative, trace-class operator on $\mathcal{H}$, by considering $d(\boldsymbol{N})$ as an operator on $\mathcal{H}_{n^{*}}$ and $\rho$ as an operator from $\mathcal{H}$ to $\mathcal{H}_{n^{*}}$. Indeed, $d(\boldsymbol{N}) \rho=$ $\sum_{n=0}^{n^{*}} \rho_{n n}(n-\bar{n})^{2}|n\rangle\langle n|$. Thus, (5) is well-defined. Moreover, since $\mathcal{H}_{n^{*}}$ is invariant under $\rho \in D_{*}$ for $n^{*} \geq n_{\max }(\rho)$, it is clear that $\operatorname{Tr}(d(\boldsymbol{N}) \rho)=\operatorname{Tr}_{\mathcal{H}_{n *}}(d(\boldsymbol{N}) \rho)$, where on the right-hand side one considers $\rho$ as an operator on the finitedimensional space $\mathcal{H}_{n^{*}}$ and the trace is taken over $\mathcal{H}_{n^{*}}$.

We have the following convergence result when $\rho_{0} \in D_{*}$ :

Theorem 1: Let $\bar{n} \in \mathbb{N}$ and $\epsilon>0$. In (2)-(4), assume that $\phi_{0} / \pi$ and $\left(\theta_{0} / \pi\right)^{2}$ are irrational numbers, and take $\phi_{R}=$ $\pi / 2-\bar{n} \phi_{0}$. Consider the closed-loop Markov process (1)(4) with $u_{k}=f\left(\rho_{k}\right)$, where the feedback law $f$ is as in (6). Then, given any initial condition $\rho_{0} \in D_{*}$, one has that $\rho_{k}$ converges almost surely towards $\bar{\rho}=|\bar{n}\rangle\langle\bar{n}|$ as $k \rightarrow \infty$.

Its proof is decomposed into two steps:

First Step. Choose $\bar{n} \in \mathbb{N}$ and $\epsilon>0$. Let $n_{0}=n_{\text {length }}\left(\rho_{0}\right)$, $r_{0}=n_{\min }\left(\rho_{0}\right)$. Then, there exists an integer $m_{0}>n_{0}+$ $r_{0}+\bar{n}+1$ (depending on $n_{0}, r_{0}, \bar{n}$ and $\epsilon$ ) such that, for all closed-loop realizations $\rho_{k}$, one has $\rho_{k} \in D_{m_{0}}$ for $k \in \mathbb{N}$.

Second Step. Choose irrational numbers $\phi_{0} / \pi$ and $\left(\theta_{0} / \pi\right)^{2}$ in (2)-(4), and take $\phi_{R}=\pi / 2-\bar{n} \phi_{0}$. In $D_{m_{0}}, V_{\epsilon}$ is a strict super-martingale: for all density operators $\rho$ in $D_{m_{0}}$, one has

$\mathbb{E}\left[V_{\epsilon}\left(\rho_{k+1}\right) \mid \rho_{k}=\rho, u_{k}=f(\rho)\right]-V_{\epsilon}(\rho)=-Q_{V_{\epsilon}}(\rho, f(\rho))$, where $Q_{V_{\epsilon}}(\rho, f(\rho)) \geq 0$, and $Q_{V_{\epsilon}}(\rho, f(\rho))=0$ if and only if $\rho=\bar{\rho}$. The almost sure convergence follows then from usual results on strict super-martingales for Markov processes with compact state spaces.

The complete proof of the two steps above is presented in Section $\mathrm{V}$ The general case where the initial condition $\rho_{0}$ is not necessarily diagonal is treated in the next subsection.

\section{B. General case}

Consider, for each $n^{*} \in \mathbb{N}$,

$$
\left.\mathbb{D}_{n^{*}}=\{\rho \in \mathbb{D}|\rho| n\rangle=0, \forall n>n^{*}\right\} \subset \mathbb{D}_{n^{*}+1},
$$

and let $\mathbb{D}_{*}=\bigcup_{n^{*} \in \mathbb{N}} \mathbb{D}_{n^{*}} \supset D_{*}$. It is clear that $\rho \in \mathbb{D}$ is in $\mathbb{D}_{n^{*}}$ if and only if $\rho=\sum_{m, n=0}^{n^{*}} \rho_{m n}|m\rangle\langle n|$. Consequently, $\mathbb{D}_{*}$ is a dense subset of $\mathbb{D}$ when $\mathbb{D}$ is endowed with the subspace topology induced from the Hilbert-Schmidt norm. Indeed, let $\mathcal{J}_{2}$ be the complex Banach space of all HilbertSchmidt operators on $\mathcal{H}$ with the Hilbert-Schmidt norm $\|B\|_{2}=\left(\sum_{m, n \in \mathbb{N}}\left|B_{m n}\right|^{2}\right)^{1 / 2}$, for $B \in \mathcal{J}_{2}$ [7], [3]. Since $\mathbb{D} \subset \mathcal{J}_{2}$ and $\rho \in \mathbb{D}_{n^{*}}$ has the form $\rho=\sum_{m, n=0}^{n^{*}} \rho_{m n}|m\rangle\langle n|$, the density property of $\mathbb{D}_{*}$ in $\mathbb{D}$ is clear.

One has that $\rho \in \mathbb{D}_{n^{*}}$ may be considered as an operator from $\mathcal{H}$ to the finite-dimensional space $\mathcal{H}_{n^{*}}$, or as a density matrix on $\mathcal{H}_{n^{*}}$. Hence, $d(\boldsymbol{N}) \rho$ is a well-defined trace-class operator on $\mathcal{H}$, by considering $d(\boldsymbol{N})$ as an operator on $\mathcal{H}_{n^{*}}$ and $\rho \in \mathbb{D}_{n^{*}}$ as an operator from $\mathcal{H}$ to $\mathcal{H}_{n^{*}}$. Indeed, $d(\boldsymbol{N}) \rho=\sum_{m, n=0}^{n^{*}} \rho_{m n}(m-\bar{n})^{2}|m\rangle\langle n|$, and it is trace-class because its range is finite-dimensional [7], [3]. Consequently, the Lyapunov function $V_{\epsilon}$ in (5), the feedback in (6) and $n_{\max }$ can be extended to $\mathbb{D}_{*}$.

Define the map $\Delta: \mathbb{D}_{*} \rightarrow D_{*} \subset \mathbb{D}_{*}$ as $\Delta \rho=$ $\sum_{n=0}^{n_{\max }(\rho)} \rho_{n n}|n\rangle\langle n|$. Note that $\Delta$ extracts the diagonal of $\rho \in \mathbb{D}_{*}$. It is easy to see that $n_{\max }(\Delta \rho)=n_{\max }(\rho)$ and $(\Delta \rho)_{n n}=\rho_{n n}, \rho \in \mathbb{D}_{*}$. Moreover, $\Delta \rho=\rho$ when $\rho \in D_{*}$. Other properties of the map $\Delta$ are given in the next result:

Proposition 2: Let $\rho \in \mathbb{D}_{*}, u \in\{-1,0,1\}, y=g, e$. Take $\alpha=\operatorname{Tr}\left(\boldsymbol{M}_{y}(u) \rho \boldsymbol{M}_{y}^{\dagger}(u)\right)$. Then:

- $\operatorname{Tr}(A \rho)=\operatorname{Tr}(A \Delta \rho)$, for every diagonal bounded operator $A: \mathcal{H} \rightarrow \mathcal{H}$;

- $V_{\epsilon}(\rho)=V_{\epsilon}(\Delta \rho)$, for $\epsilon>0$;

- $\alpha^{-1} \boldsymbol{M}_{y}(u) \rho \boldsymbol{M}_{y}^{\dagger}(u)$ belongs to $\mathbb{D}_{*}$ with $\Delta\left(\alpha^{-1} \boldsymbol{M}_{y}(u) \rho \boldsymbol{M}_{y}^{\dagger}(u)\right)=\alpha^{-1} \boldsymbol{M}_{y}(u)(\Delta \rho) \boldsymbol{M}_{y}^{\dagger}(u)$;

- $\left[\boldsymbol{M}_{y}(u)(\Delta \rho) \boldsymbol{M}_{y}^{\dagger}(u)\right]_{n n}=\left[\boldsymbol{M}_{y}(u) \rho \boldsymbol{M}_{y}^{\dagger}(u)\right]_{n n}$, for all $n \in \mathbb{N}$. In particular, $\alpha=\operatorname{Tr}\left(M_{y}(u)(\Delta \rho) M_{y}^{\dagger}(u)\right)$. Proof: See Appendix G

Now, let $\epsilon>0$ and $\bar{\rho}=|\bar{n}\rangle\langle\bar{n}|$, where $\bar{n} \in \mathbb{N}$. Assume that $\rho_{0} \in \mathbb{D}_{*}$. Let $\rho_{k}, k \in \mathbb{N}$, be the corresponding closed-loop trajectory for a fixed realization of (1)-(4) with feedback $u_{k}=f\left(\rho_{k}\right)$, where $f$ is as in (6). It is immediate from the proposition above that:

- $\rho_{k} \in \mathbb{D}_{*}$, for $k \in \mathbb{N}$;

- $\Delta \rho_{k} \in D_{*}, k \in \mathbb{N}$, is the corresponding closed-loop trajectory of (1)-(4) for the initial condition $\Delta \rho_{0}$, the same realization (and with the same transition probabilities $p_{e, k}$ and $\left.p_{g, k}\right)$, as well as the same feedback $u_{k}=f\left(\rho_{k}\right)=f\left(\Delta \rho_{k}\right)$;

- $\operatorname{Tr}\left(|n\rangle\langle n| \rho_{k}\right)=\operatorname{Tr}\left(|n\rangle\langle n| \Delta \rho_{k}\right)$, for any $n \in \mathbb{N}$.

From these arguments, Theorem 1 and the fact that $\Delta \bar{\rho}=$ $\bar{\rho}$, one immediately obtains the following generic solution to the control problem, that is, when the initial condition $\rho_{0}$ belongs to the dense subset $\mathbb{D}_{*}$ of $\mathbb{D}$ :

Theorem 2: Let $\bar{n} \in \mathbb{N}$ and $\epsilon>0$. In (2)-(4), assume that $\phi_{0} / \pi$ and $\left(\theta_{0} / \pi\right)^{2}$ are irrational numbers, and take $\phi_{R}=$ $\pi / 2-\bar{n} \phi_{0}$. Consider the closed-loop Markov process (1) (4) with $u_{k}=f\left(\rho_{k}\right)$, where the feedback law $f$ is as in (6). Then, given any initial condition $\rho_{0} \in \mathbb{D}_{*}$, one has that $\rho_{k}$ converges almost surely towards $\bar{\rho}=|\bar{n}\rangle\langle\bar{n}|$ as $k \rightarrow \infty$. 


\section{SIMULATION RESULTS}

This section presents the closed-loop simulation results concerning the application of Theorem 2 above to the ideal Markov process (1)-(4). The quantum experimental results exhibited in [8] used the following control parameter values in (2)-(4): $\phi_{0} / \pi=0.252$ and $\theta_{0} / \pi \approx 2 / \sqrt{\bar{n}+1}$. However, according to the assumptions in Theorem 2 and $\left(\theta_{0} / \pi\right)^{2}$ should be irrational numbers. Hence, here one chooses $\phi_{0} / 3.14=0.252$ and $\theta_{0} / 3.14=2 / \sqrt{\bar{n}+1}$. One takes $\rho_{0}=\sum_{n=0}^{15}|n\rangle\langle n| / 16 \in \mathbb{D}_{*}$ as the initial condition, $\bar{n}=10$ for the goal Fock state $\bar{\rho}=|\bar{n}\rangle\langle\bar{n}|$, and $\epsilon=10^{3}$ as the gain for the feedback $u_{k}=f\left(\rho_{k}\right)$ in (5)-(6). Figure 1 exhibits the simulation results for one closed-loop realization with such choices and a final sample step of 120. It shows: the dynamics of the populations of $\rho_{k}$ (top), the controls $u_{k}$ (middle) and the simulated outcomes $y_{k}$ (bottom). The populations of $\rho_{k}$ correspond to the following observables: $A_{1}=\sum_{n=0}^{\bar{n}-1}|n\rangle\left\langle n\left|(n<\bar{n}), A_{2}=\right| \bar{n}\right\rangle\langle\bar{n}|(n=\bar{n})$, $A_{3}=\sum_{n>\bar{n}}|n\rangle\langle n|(n>\bar{n})$. Therefore, one sees from the dynamics of the populations that $\rho_{k}$ converges to $\bar{\rho}$ as $k \rightarrow \infty$, which is in accordance with Theorem 2] Note that $\left\langle\bar{n}\left|\rho_{k}\right| \bar{n}\right\rangle \approx 1$ and $u_{k}=0$ for all $k>45$.

Recall that Theorem 2 assumes that $\epsilon>0$. In order to further analyze the performance of the Lyapunov-based feedback law here proposed, we now make a comparison with the one used experimentally in [8], which corresponds to take $\epsilon=0$ in (5), i.e. to disregard the term $-\epsilon \sum_{n \in \mathbb{N}} \rho_{n n}^{2}$. Figure 2 presents the simulation results for one closed-loop realization of such case. The control parameters, $\rho_{0}$ and $\bar{n}=$ 10 are the same as above. Note that $\left\langle\bar{n}\left|\rho_{k}\right| \bar{n}\right\rangle \approx 1$ and $u_{k}=0$ for all $k>78$. In order to make a comparison in terms of the speed of convergence, define the settling time $k_{s}$ to be the smallest $\widetilde{k} \in \mathbb{N}$ such that $\left\langle\bar{n}\left|\rho_{k}\right| \bar{n}\right\rangle>0.9$ for all $k \geq \widetilde{k}$. One has $k_{s}=45$ for the case $\epsilon=10^{3}$ above, and $k_{s}=78$ for $\epsilon=0$. Therefore, in the two realizations here simulated, the choice of $\epsilon=10^{3}$ reduced the settling time $k_{s}$ by nearly $42 \%$ with respect to $\epsilon=0$. This behavior is typical on an average basis, thereby justifying the term $-\epsilon \sum_{n \in \mathbb{N}} \rho_{n n}^{2}$ in (5). Table I] shows the average value $\bar{k}_{s}$ and the standard deviation $\sigma$ of $k_{s}$ for $\epsilon \in\left\{0,0.1,1,10,10^{2}, 10^{3}, 10^{4}, 10^{5}\right\}$, where a total of 5000 realizations were simulated for each $\epsilon$. Notice that when $\epsilon$ is relatively large or relatively small in comparison to $\epsilon=10^{3}$, the average settling time $\bar{k}_{s}$ deteriorated. Furthermore, although for $\epsilon=10^{5}$ one has that $\bar{k}_{s}$ increased by nearly $22 \%$ in comparison to $\epsilon=10^{3}$, the standard deviation $\sigma$ decreased by nearly $62 \%$. Computer simulations have suggested that a choice of $\epsilon>0$ which may perhaps significantly improve $\bar{k}_{s}$ generally depends on the initial condition $\rho_{0}$ and on the goal Fock state $\bar{\rho}=|\bar{n}\rangle\langle\bar{n}|$, and it has to be determined heuristically.

\section{PROOF OF THEOREM11(DIAGONAL CASE)}

\section{Proof of the First Step:}

Let $\epsilon>0$. Define $V: D_{*} \rightarrow \mathbb{R}$ and $W: D_{*} \rightarrow \mathbb{R}$ as

$$
V(\rho)=\operatorname{Tr}(d(\boldsymbol{N}) \rho), \quad W(\rho)=-\sum_{n \in \mathbb{N}} \rho_{n n}^{2},
$$
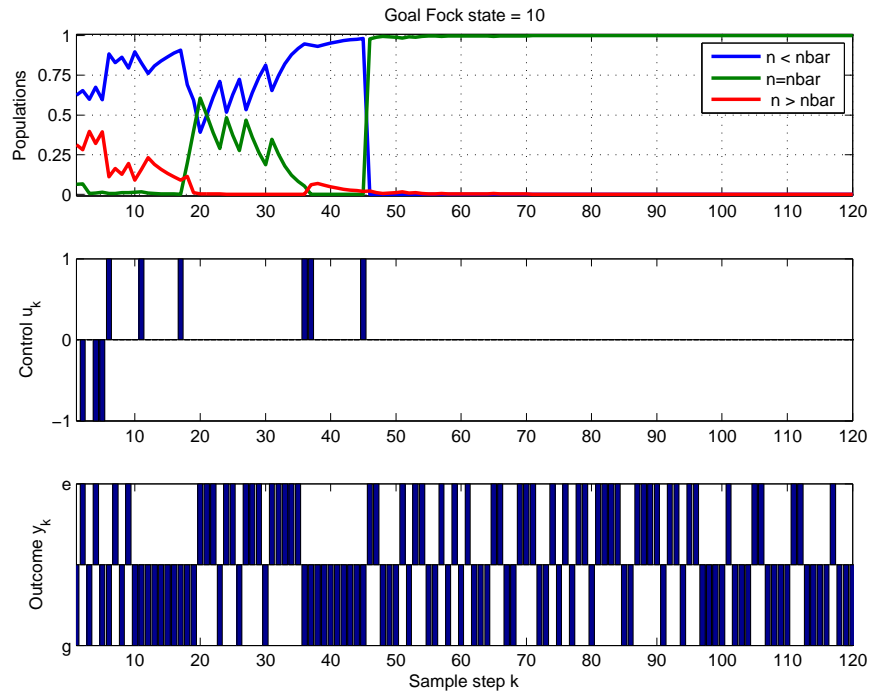

Fig. 1. Simulation of one closed-loop realization with gain $\epsilon=10^{3}$ : convergence of $\rho_{k}$ towards $\bar{\rho}$ (top), controls $u_{k}$ (middle), and outcomes $y_{k}$ (bottom). Notice that $\left\langle\bar{n}\left|\rho_{k}\right| \bar{n}\right\rangle \approx 1$ and $u_{k}=0$ for all $k>45$.
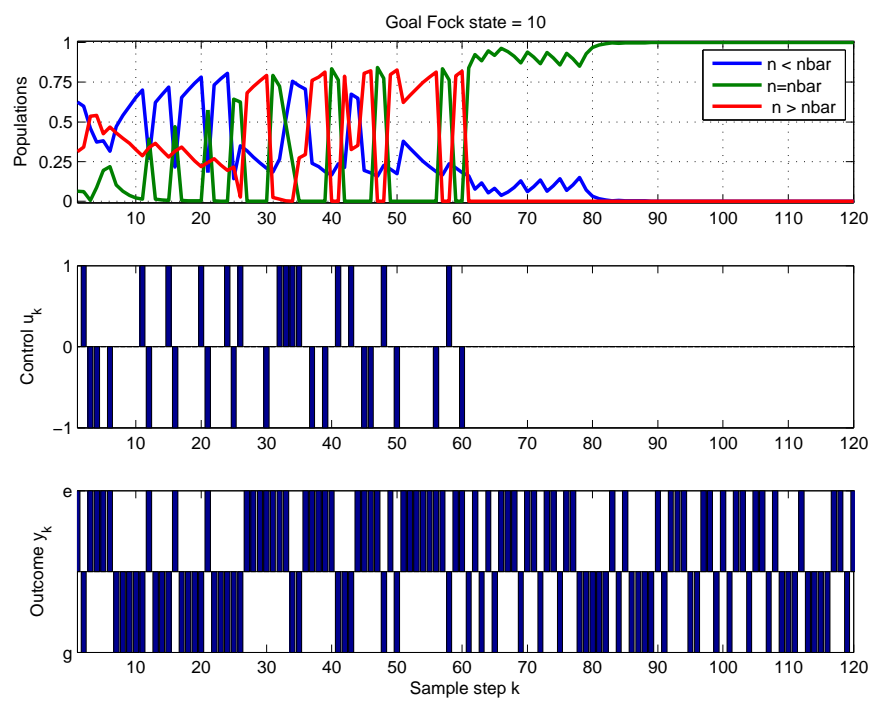

Fig. 2. Simulation of one closed-loop realization with gain $\epsilon=0$ : convergence of $\rho_{k}$ towards $\bar{\rho}$ (top), controls $u_{k}$ (middle), and outcomes $y_{k}$ (bottom). Notice that $\left\langle\bar{n}\left|\rho_{k}\right| \bar{n}\right\rangle \approx 1$ and $u_{k}=0$ for all $k>78$.

TABLE I

AVERAGE SETTLING TIME $\bar{k}_{s}$ AND STANDARD DEVIATION $\sigma$ AS A FUNCTION OF THE GAINS $\epsilon$, CONSIDERING 5000 REALIZATIONS

\begin{tabular}{|l|l|l|l|}
\hline$\epsilon=0$ & $\epsilon=0.1$ & $\epsilon=1$ & $\epsilon=10$ \\
$\bar{k}_{s}=79.94$ & $\bar{k}_{s}=79.95$ & $\bar{k}_{s}=81.24$ & $\bar{k}_{s}=71.33$ \\
$\sigma=164.97$ & $\sigma=166.61$ & $\sigma=174.29$ & $\sigma=150.95$ \\
\hline \hline$\epsilon=10^{2}$ & $\epsilon=10^{3}$ & $\epsilon=10^{4}$ & $\epsilon=10^{5}$ \\
$\bar{k}_{s}=60.41$ & $\bar{k}_{s}=44.18$ & $\bar{k}_{s}=47.05$ & $\bar{k}_{s}=53.77$ \\
$\sigma=119.39$ & $\sigma=44.12$ & $\sigma=37.37$ & $\sigma=16.84$ \\
\hline
\end{tabular}

respectively. Note that $V_{\epsilon}=V+\epsilon W$. Define:
- $Q_{W}(\rho, u)=W(\rho)-\mathbb{E}\left[W\left(\rho_{k+1}\right) \mid \rho_{k}=\rho, u_{k}=u\right]$,
- $Q_{V}(\rho, u)=V(\rho)-\mathbb{E}\left[V\left(\rho_{k+1}\right) \mid \rho_{k}=\rho, u_{k}=u\right]$,
- $Q_{V_{\epsilon}}(\rho, u)=V_{\epsilon}(\rho)-\mathbb{E}\left[V_{\epsilon}\left(\rho_{k+1}\right) \mid \rho_{k}=\rho, u_{k}=u\right]$, 
for $\rho \in D_{*}$ and $u \in\{-1,0,1\}$. The proof of Theorem 1 is a straightforward consequence of the next proposition:

Proposition 3: Let $\epsilon>0$ and $n_{0}, r_{0}, \bar{n} \in \mathbb{N}$. There exists an integer $m_{0}>n_{0}+r_{0}+\bar{n}+1$ (depending on $\epsilon, n_{0}, r_{0}, \bar{n}$ ) such that, for each $\rho \in D_{*}$ with $n_{\text {length }}(\rho) \leq n_{0}$, if $n_{\max }(\rho)=m_{0}$, then

$$
Q_{V_{\epsilon}}(\rho,-1)>\max \left\{Q_{V_{\epsilon}}(\rho, 0), Q_{V_{\epsilon}}(\rho,+1)\right\} .
$$

In fact, given $\rho_{0} \in D_{*}$, let $n_{0}=n_{\text {length }}\left(\rho_{0}\right)$ and $r_{0}=n_{\min }\left(\rho_{0}\right)$. Note that $n_{\max }\left(\rho_{0}\right)=n_{0}+r_{0}<m_{0}$. By Proposition $1 \rho_{k} \in D_{*}$ with $n_{\text {length }}\left(\rho_{k}\right) \leq n_{0}$, for all $k \in \mathbb{N}$. Since $u=f(\rho)$ maximizes $Q_{V_{\epsilon}}(\rho, f(\rho))$, Proposition 3 implies that when $n_{\max }\left(\rho_{k}\right)=m_{0}$ for some $k \in \mathbb{N}$, then the input $u_{k}$ will be always be equal to -1 , and hence Proposition 1 ensures that $n_{\max }\left(\rho_{k+1}\right) \leq n_{\max }\left(\rho_{k}\right)=m_{0}$. Therefore, $n_{\max }\left(\rho_{k}\right) \leq m_{0}, k \in \mathbb{N}$, showing the First Step.

The following two lemmas are instrumental for showing Proposition 3 Their proofs are given in Appendix D and Appendix E, respectively.

Lemma 1: Given an arbitrary nonzero $\theta_{0} \in \mathbb{R}$, fix any $a \in \mathbb{R}$ such that $0<a<1 / 2$. For all nonzero $N_{0}, N \in \mathbb{N}$, there exists an integer $\bar{N}>N$ big enough such that,

$$
0<1 / 2-a \leq \sin ^{2}\left(\frac{\theta_{0}}{2} \sqrt{n}\right) \leq 1 / 2+a
$$

for $n=\bar{N}, \bar{N}+1, \ldots, \bar{N}+N_{0}-1$.

Lemma 2: Let $\rho \in D_{*}$. Then:

- $\left|Q_{W}(\rho, u)\right| \leq 1, \quad$ for each $u \in\{-1,0,1\}$;

- $Q_{V}(\rho, 0)=0$;

- $Q_{V}(\rho,+1)=-\sum_{n \in \mathbb{N}} \rho_{n n}[2(n-\bar{n})+1] \sin ^{2}\left(\frac{\theta_{0}}{2} \sqrt{n+1}\right)$;

- $Q_{V}(\rho,-1)=\sum_{n \in \mathbb{N}} \rho_{n n}[2(n-\bar{n})-1] \sin ^{2}\left(\frac{\theta_{0}}{2} \sqrt{n}\right)$.

The proof of Proposition 3 is shown in the sequel. Proof: Let $\epsilon>0$ and $n_{0}, r_{0}, \bar{n} \in \mathbb{N}$. One has to show that there exists $m_{0}>n_{0}+r_{0}+\bar{n}+1$ such that, if $\rho \in D_{*}$ with $n_{\text {length }}(\rho) \leq n_{0}$, then $u=-1$ always maximizes $Q_{V_{\epsilon}}(\rho, u)$ whenever $n_{\max }(\rho)=m_{0}$. From Lemma 2 and the fact that $Q_{V_{\epsilon}}=Q_{V}+\epsilon Q_{W}$, to complete the proof it suffices to show that:

- If $\rho \in D_{*}$ is such that $n_{\text {length }}(\rho) \leq n_{0}$ and $n_{\max }(\rho) \geq$ $n_{0}+\bar{n}$, then $Q_{V}(\rho,+1) \leq 0$;

- There exists $m_{0}>n_{0}+r_{0}+\bar{n}+1$ such that $Q_{V}(\rho,-1)>2 \epsilon$, whenever $\rho \in D_{*}$ is such that $n_{\text {length }}(\rho) \leq n_{0}$ and $n_{\max }(\rho)=m_{0}$.

Note that

$Q_{V}(\rho,+1)=-\sum_{n=n_{\min }(\rho)}^{n_{\max }(\rho)} \rho_{n n}[2(n-\bar{n})+1] \sin ^{2}\left(\frac{\theta_{0}}{2} \sqrt{n+1}\right)$,

for any $\rho \in D_{*}$. Thus, if $n_{\text {length }}(\rho) \leq n_{0}$ and $n_{\max }(\rho) \geq$ $\bar{n}+n_{0}$, then $n_{\min }(\rho) \geq \bar{n}$, and hence the first claim is shown.

Now, fix $0<a<1 / 2$ and let $N \geq \frac{1}{2}\left[\frac{2 \epsilon}{1 / 2-a}+2 \bar{n}+1\right]$. Applying Lemma 1 for $N_{0}=n_{0}+r_{0}+1$ and such choice of $N$, one gets $\bar{N}>N$ in which $0<1 / 2-a \leq \sin ^{2}\left(\frac{\theta_{0}}{2} \sqrt{n}\right)$,

${ }^{4}$ As $N$ is an integer, it follows that $N \geq \bar{n}+1$. for $n=\bar{N}, \bar{N}+1, \ldots, \bar{N}+n_{0}+r_{0}$. Take $m_{0}=\bar{N}+n_{0}+r_{0}$. Let $\rho \in D_{*}$ with $n_{\text {length }}(\rho) \leq n_{0}$ and $n_{\max }(\rho)=m_{0}$. Note that $m_{0}>n_{0}+r_{0}+\bar{n}+1$ and $n_{\min }(\rho) \geq \bar{N}+r_{0}$. From Lemma 2 and the inequality above for $1 / 2-a$, one obtains

$$
\begin{aligned}
& Q_{V}(\rho,-1)=\sum_{n=n_{\min }(\rho)}^{m_{0}} \rho_{n n}[2(n-\bar{n})-1] \sin ^{2}\left(\frac{\theta_{0}}{2} \sqrt{n}\right) \\
& \geq \sum_{n=n_{\min }(\rho)}^{m_{0}} \rho_{n n}[2(n-\bar{n})-1](1 / 2-a) \\
& \geq \sum_{n=n_{\min }(\rho)}^{m_{0}} \rho_{n n}[2(\bar{N}-\bar{n})-1](1 / 2-a) \\
& =[2(\bar{N}-\bar{n})-1](1 / 2-a) \sum_{n=n_{\min }(\rho)}^{m_{0}} \rho_{n n} .
\end{aligned}
$$

Using the fact that $\sum_{n=n_{\min }(\rho)}^{m_{0}} \rho_{n n}=1$ and $\bar{N}>$ $\frac{1}{2}\left[\frac{2 \epsilon}{1 / 2-a}+2 \bar{n}+1\right]$, one shows the second claim, thereby completing the proof of Proposition 3 .

\section{Proof of the Second Step:}

Let $\epsilon>0$. Recall that, by definition, $Q_{V_{\epsilon}}=Q_{V}+\epsilon Q_{W}$. Using the same notation of the First Step, the central idea of the proof is to show that, given $\rho \in D_{m_{0}}$, one has that $Q_{V_{\epsilon}}(\rho, f(\rho)) \geq 0$, and that $Q_{V_{\epsilon}}(\rho, f(\rho))=0$ if and only if $\rho=\bar{\rho}$. The following lemma is instrumental for the proof of such property. Its proof is presented in Appendix $\mathrm{F}$

Lemma 3: Assume that $\phi_{0} / \pi$ is an irrational number in (2), and take $\phi_{R}=\pi / 2-\bar{n} \phi_{0}$, where $\bar{n} \in \mathbb{N}$. Let $\rho \in D_{*}$. Then:

- $Q_{W}(\rho, 0) \geq 0$, and $Q_{W}(\rho, 0)=0$ if and only if $\rho=$ $|n\rangle\langle n|$ for some $n \in \mathbb{N}$;

- $Q_{W}(\rho,+1)=Q_{W}(\rho,-1)=0$ whenever $\rho=|n\rangle\langle n|$ for some $n \in \mathbb{N}$.

One has that $m_{0}>\bar{n}$, and $\left(\theta_{0} / \pi\right)^{2}$ is an irrational number by assumption. Recall that $\sin ^{2}(x)=0$ if and only if $x=\ell \pi$, where $\ell$ is an integer. First we show that $Q_{V_{\epsilon}}(\bar{\rho}, f(\bar{\rho}))=$ 0 . By Lemma 2] $Q_{V}(\bar{\rho},+1)=-\sin ^{2}\left(\frac{\theta_{0}}{2} \sqrt{\bar{n}+1}\right)<0$; $Q_{V}(\bar{\rho},-1)=-\sin ^{2}\left(\frac{\theta_{0}}{2} \sqrt{\bar{n}}\right)<0$ when $\bar{n}>0$, and $Q_{V}(\bar{\rho},-1)=0$ when $\bar{n}=0$; and $Q_{V}(\bar{\rho}, 0)=0$. As $Q_{W}(\bar{\rho}, u)=0$, for $u \in\{-1,0,1\}$, and $u=f(\bar{\rho})$ maximizes $Q_{V_{\epsilon}}(\bar{\rho}, u)$, one has that $Q_{V_{\epsilon}}(\bar{\rho}, f(\bar{\rho}))=0$.

Now, let $\rho \in D_{m_{0}} \subset D_{*}$. Since $u=f(\rho)$ maximizes $Q_{V_{\epsilon}}(\rho, u)$, it follows that

$$
Q_{V_{\epsilon}}(\rho, f(\rho)) \geq Q_{V}(\rho, 0)+\epsilon Q_{W}(\rho, 0)=\epsilon Q_{W}(\rho, 0) \geq 0 .
$$

Suppose $Q_{V_{\epsilon}}(\rho, f(\rho))=0$. Hence, $Q_{W}(\rho, 0)=0$, and so $\rho=|n\rangle\langle n|$ for some $n \in\left\{0,1, \ldots, m_{0}\right\}$. It suffices to show that $\left.Q_{V_{\epsilon}}(\rho, f(\rho))\right|_{|n\rangle\langle n|}>0$ for $n \in\left\{0,1, \ldots, m_{0}\right\}$ with $n \neq \bar{n}$. Assume that $n>\bar{n}$. It is clear that $u=f(|n\rangle\langle n|)=$ -1 and $\left.Q_{V_{\epsilon}}(\rho, f(\rho))\right|_{|n\rangle\langle n|}=[2(n-\bar{n})-1] \sin ^{2}\left(\frac{\theta_{0}}{2} \sqrt{n}\right)>0$. Assume now that $n<\bar{n}$. Then, $u=f(|n\rangle\langle n|)=+1$ and $\left.Q_{V_{\epsilon}}(\rho, f(\rho))\right|_{|n\rangle\langle n|}=-[2(n-\bar{n})+1] \sin ^{2}\left(\frac{\theta_{0}}{2} \sqrt{n+1}\right)>0$. This completes the proof of the referred property.

The remaining part of the proof of the Second Step is a straightforward consequence of the standard stochastic convergence result below: 
Theorem 3: [5, Theorem 1, p. 195] Let $\Omega$ be a probability space and let $W$ be a measurable space. Consider that $X_{k}: \Omega \rightarrow W, k \in \mathbb{N}$, is a Markov chain with respect to the natural filtration. Let $Q: W \rightarrow \mathbb{R}$ and $V: W \rightarrow \mathbb{R}$ be measurable non-negative functions with $V\left(X_{k}\right)$ integrable for all $k \in \mathbb{N}$. If $\mathbb{E}\left[V\left(X_{k+1}\right) \mid X_{k}\right]-V\left(X_{k}\right)=-Q\left(X_{k}\right)$, for $k \in \mathbb{N}$, then $\lim _{k \rightarrow \infty} Q\left(X_{k}\right)=0$ almost surely.

Indeed, let $\mathcal{J}_{1}$ be the complex Banach space of all traceclass operators on $\mathcal{H}$ with the trace norm $\|\cdot\|_{1}$, that is, $\|B\|_{1}=\operatorname{Tr}(|B|)$, where $|B| \triangleq \sqrt{B^{\dagger} B}$, for $B \in \mathcal{J}_{1}$. Recall that $\|B\| \leq\|B\|_{1}$ and $|\operatorname{Tr}(A B)| \leq\|A\|\|B\|_{1}$, for every $B \in \mathcal{J}_{1}$ and each bounded operator $A$ : $\mathcal{H} \rightarrow \mathcal{H}$, where $\|\cdot\|$ is the usual operator norm (sup norm of bounded operators) [7], [3]. Consider the subspace topology on $D_{m_{0}}$ with respect to $\mathcal{J}_{1}$. One has that the closed-loop trajectory $\rho_{k}, k \in \mathbb{N}$, is a Markov chain with phase space $D_{m_{0}}$ (with respect to the natural filtration and the Borel algebra on $D_{m_{0}}$ ). It is clear that $D_{m_{0}}$ is compact, and that $Q_{\epsilon}$ and $V_{\epsilon}-\alpha_{\epsilon}$ are non-negative and continuous on $D_{m_{0}}$, for all $\epsilon>0$, where $\alpha_{\epsilon} \triangleq \min _{\rho \in D_{m_{0}}} V_{\epsilon}(\rho)$. The theorem above implies that $\rho_{k}$ converges almost surely towards $\bar{\rho}$ as $k \rightarrow \infty$ (with respect to the trace norm). This completes the proof of Theorem 1

\section{CONCLUDING REMARKS}

This paper provided a convergence analysis of Fock states stabilization via single-photon corrections under an ideal setup, that is, assuming perfect measurement detection and no control delays. In terms of convergence speed, the simulation results here presented have justified the inclusion of the term $-\epsilon \sum_{n \in \mathbb{N}} \rho_{n n}^{2}$ in the Lyapunov-based feedback law (5)-(6). It is straightforward to verify that the convergence analysis developed in this paper remains valid for: (i) any other function $d(n)$ in (5) satisfying $d(\bar{n})=0, d(n)$ is increasing for $n>\bar{n}$ and $d(n)$ is decreasing for $n<\bar{n}$; and (ii) $\epsilon>0$ dependent on $n$, that is, to take the term $-\sum_{n \in \mathbb{N}} \epsilon_{n} \rho_{n n}^{2}$. However, it is an open problem how to choose the function $d(n)$ and the gains $\epsilon_{n}>0$ so as to achieve the best convergence speed.

Finally, the feedback law used in [8], which corresponds to $\epsilon=0$, was tailored for an experimental set-up with measurement imperfections and control delays. The convergence analysis of such realistic situation will be investigated in the future.

\section{ACKNOWLEDGMENTS}

The authors are indebted I. Dotsenko, M. Brune and J. M. Raimond for valuable discussions on the experimental feedback scheme.

\section{APPENDIX}

\section{A. Basic properties of the operators $\boldsymbol{N}, \boldsymbol{a}$ and $\boldsymbol{a}^{\dagger}$}

Fix $n^{*} \in \mathbb{N}$ and let $\mathcal{H}_{n^{*}}=\operatorname{span}\left\{|0\rangle, \ldots,\left|n^{*}\right\rangle\right\}$. Consider the (linear) operators $N: \mathcal{H}_{n^{*}} \rightarrow \mathcal{H}_{n^{*}}, \boldsymbol{a}: \mathcal{H}_{n^{*}} \rightarrow$ $\mathcal{H}_{n^{*}-1} \subset \mathcal{H}_{n^{*}}, \boldsymbol{a}^{\dagger}: \mathcal{H}_{n^{*}} \rightarrow \mathcal{H}_{n^{*}+1}$ defined respectively as $\boldsymbol{N}|n\rangle=n|n\rangle, \boldsymbol{a}|0\rangle=0, \boldsymbol{a}|n\rangle=\sqrt{n}|n-1\rangle$ for $n \geq 1$,

\footnotetext{
${ }^{5}$ One also recalls that if $A$ is a bounded operator on $\mathcal{H}$ and $B \in \mathcal{J}_{1}$, then $A B, B A \in \mathcal{J}_{1}$ with $\operatorname{Tr}(A B)=\operatorname{Tr}(B A)$.
}

$\boldsymbol{a}^{\dagger}|n\rangle=\sqrt{n+1}|n+1\rangle$. Note that these operators cannot be extended to $\mathcal{H}$. Let $f: \mathbb{N} \rightarrow \mathbb{R}$ be a function. Define the operator $f(\boldsymbol{N}): \mathcal{H}_{n^{*}} \rightarrow \mathcal{H}_{n^{*}}$ by $f(\boldsymbol{N})|n\rangle=f(n)|n\rangle$, for each $n=0, \ldots, n^{*}$. It is clear that $f(\boldsymbol{N})$ can be extented to $\mathcal{H}$ whenever $f$ is a bounded function. Given $f: \mathbb{N} \rightarrow \mathbb{R}$ and an integer $m$, one defines $g: \mathbb{N} \rightarrow \mathbb{R}$ as: $g(n)=f(n+m)$, when $n+m \geq 0$; and $g(n)=0$, when $n+m<0$. One abuses notation letting $f(\boldsymbol{N}+m)$ stand for $g(\boldsymbol{N})$. Given two functions $f, g: \mathbb{N} \rightarrow \mathbb{R}$, it is clear that $f(\boldsymbol{N}) g(\boldsymbol{N})=g(\boldsymbol{N}) f(\boldsymbol{N})=(f g)(\boldsymbol{N})$ and $(f+g)(\boldsymbol{N})=f(\boldsymbol{N})+g(\boldsymbol{N})$. Furthermore: $\boldsymbol{a a}^{\dagger}=\boldsymbol{N}+\mathbf{I}$, $\boldsymbol{a}^{\dagger} \boldsymbol{a}=\boldsymbol{N}, \boldsymbol{a} f(\boldsymbol{N})=f(\boldsymbol{N}+1) \boldsymbol{a}, \boldsymbol{a}^{\dagger} f(\boldsymbol{N})=f(\boldsymbol{N}-1) \boldsymbol{a}^{\dagger}$.

\section{B. Proof of Proposition 1}

Fix any $\rho \in D_{*}$ and let $n \in \mathbb{N}$. In particular, $\rho|n\rangle=$ $\rho_{n n}|n\rangle$. It then follows from (2)-(4) that:

$$
\begin{aligned}
& \boldsymbol{M}_{g}(0) \rho \boldsymbol{M}_{g}^{\dagger}(0)|n\rangle=\rho_{n n} \cos ^{2}\left(\frac{\phi_{0} n+\phi_{R}}{2}\right)|n\rangle, \\
& \boldsymbol{M}_{e}(0) \rho \boldsymbol{M}_{e}^{\dagger}(0)|n\rangle=\rho_{n n} \sin ^{2}\left(\frac{\phi_{0} n+\phi_{R}}{2}\right)|n\rangle, \\
& \boldsymbol{M}_{g}(+1) \rho \boldsymbol{M}_{g}^{\dagger}(+1)|n\rangle=\left\{\begin{array}{l}
0, \text { for } n=0, \\
\rho_{n-1, n-1} \sin ^{2}\left(\frac{\theta_{0}}{2} \sqrt{n}\right)|n\rangle, n \geq 1,
\end{array}\right. \\
& \boldsymbol{M}_{e}(+1) \rho \boldsymbol{M}_{e}^{\dagger}(+1)|n\rangle=\rho_{n n} \cos ^{2}\left(\frac{\theta_{0}}{2} \sqrt{n+1}\right)|n\rangle, \\
& \boldsymbol{M}_{g}(-1) \rho \boldsymbol{M}_{g}^{\dagger}(-1)|n\rangle=\rho_{n n} \cos ^{2}\left(\frac{\theta_{0}}{2} \sqrt{n}\right)|n\rangle, \\
& \boldsymbol{M}_{e}(-1) \rho \boldsymbol{M}_{e}^{\dagger}(-1)|n\rangle=\rho_{n+1, n+1} \sin ^{2}\left(\frac{\theta_{0}}{2} \sqrt{n+1}\right)|n\rangle .
\end{aligned}
$$

Therefore:

$$
\begin{aligned}
& \boldsymbol{M}_{g}(0) \rho \boldsymbol{M}_{g}^{\dagger}(0)=\sum_{n=n_{\min }(\rho)}^{n_{\max }(\rho)} \rho_{n n} \cos ^{2}\left(\frac{\phi_{0} n+\phi_{R}}{2}\right)|n\rangle\langle n|, \\
& \boldsymbol{M}_{e}(0) \rho \boldsymbol{M}_{e}^{\dagger}(0)=\sum_{n=n_{\min }(\rho)}^{n_{\max }(\rho)} \rho_{n n} \sin ^{2}\left(\frac{\phi_{0} n+\phi_{R}}{2}\right)|n\rangle\langle n|, \\
& \boldsymbol{M}_{g}(+1) \rho \boldsymbol{M}_{g}^{\dagger}(+1)=\sum_{n=n_{\min }(\rho)+1}^{n_{\max }(\rho)+1} \rho_{n-1, n-1} \sin ^{2}\left(\frac{\theta_{0}}{2} \sqrt{n}\right)|n\rangle\langle n|, \\
& \boldsymbol{M}_{e}(+1) \rho \boldsymbol{M}_{e}^{\dagger}(+1)=\sum_{n=n_{\min }(\rho)}^{n_{\max }(\rho)} \rho_{n n} \cos ^{2}\left(\frac{\theta_{0}}{2} \sqrt{n+1}\right)|n\rangle\langle n|, \\
& \boldsymbol{M}_{g}(-1) \rho \boldsymbol{M}_{g}^{\dagger}(-1)=\sum_{n=n_{\min }(\rho)}^{n_{\max }(\rho)} \rho_{n n} \cos ^{2}\left(\frac{\theta_{0}}{2} \sqrt{n}\right)|n\rangle\langle n|, \\
& \boldsymbol{M}_{e}(-1) \rho \boldsymbol{M}_{e}^{\dagger}(-1)=\sum_{n=\max \left\{0, n_{\min }(\rho)-1\right\}}^{n_{\max }(\rho)-1} \rho_{n+1, n+1} \sin ^{2}\left(\frac{\theta_{0}}{2} \sqrt{n+1}\right)|n\rangle\langle n| .
\end{aligned}
$$

By assumption, $\rho_{0} \in D_{*}$. Then, (1), (8)-(13) above and induction on $k$ show the assertions in Proposition 1

\section{Computation of $Q_{V}(\rho, u)$}

Fix any $\rho \in D_{*}$ and $\bar{n} \in \mathbb{N}$. Recall that $V(\rho)=$ $\operatorname{Tr}(d(\boldsymbol{N}) \rho)$, where $d: \mathbb{N} \rightarrow \mathbb{R}$ be given by $d(n)=(n-\bar{n})^{2}$. Note that (1) implies that, for each $u \in\{-1,0,1\}$,

$$
\begin{aligned}
& \mathbb{E}\left[V\left(\rho_{k+1}\right) \mid \rho_{k}=\rho, u_{k}=u\right] \\
& =\operatorname{Tr}\left(d(\boldsymbol{N}) \boldsymbol{M}_{g}(u) \rho \boldsymbol{M}_{g}^{\dagger}(u)\right)+\operatorname{Tr}\left(d(\boldsymbol{N}) \boldsymbol{M}_{e}(u) \rho \boldsymbol{M}_{e}^{\dagger}(u)\right) .
\end{aligned}
$$


Take $u=0$. From (8)-(9) in Appendix B, one has

$$
\begin{aligned}
& \mathbb{E}\left[V\left(\rho_{k+1}\right) \mid \rho_{k}=\rho, u_{k}=0\right] \\
& =\operatorname{Tr}\left(d(\boldsymbol{N}) \boldsymbol{M}_{g}(0) \rho \boldsymbol{M}_{g}^{\dagger}(0)\right)+\operatorname{Tr}\left(d(\boldsymbol{N}) \boldsymbol{M}_{e}(0) \rho \boldsymbol{M}_{e}^{\dagger}(0)\right) \\
& =\operatorname{Tr}\left(d(\boldsymbol{N})\left[\boldsymbol{M}_{g}(0) \rho \boldsymbol{M}_{g}^{\dagger}(0)+\boldsymbol{M}_{e}(0) \rho \boldsymbol{M}_{e}^{\dagger}(0)\right]\right) \\
& =\operatorname{Tr}(d(\boldsymbol{N}) \rho)=V(\rho) .
\end{aligned}
$$

In particular,

$$
Q_{V}(\rho, 0)=0 .
$$

Now, take $u=+1$. Then, (14) above and (10)-(11) in Appendix B provide that

$$
\begin{aligned}
& \mathbb{E}\left[V\left(\rho_{k+1}\right) \mid \rho_{k}=\rho, u_{k}=+1\right] \\
& =\operatorname{Tr}\left(\sin ^{2}\left(\frac{\theta_{0}}{2} \sqrt{\boldsymbol{N}+1}\right) d(\boldsymbol{N}+1) \rho\right) \\
& +\operatorname{Tr}\left(\cos ^{2}\left(\frac{\theta_{0}}{2} \sqrt{\boldsymbol{N}+1}\right) d(\boldsymbol{N}) \rho\right) .
\end{aligned}
$$

By summing and subtracting $\operatorname{Tr}\left(\sin ^{2}\left(\frac{\theta_{0}}{2} \sqrt{\boldsymbol{N}+1}\right) d(\boldsymbol{N}) \rho\right)$,

$$
\begin{aligned}
& \mathbb{E}\left[V\left(\rho_{k+1}\right) \mid \rho_{k}=\rho, u_{k}=+1\right] \\
& =\operatorname{Tr}(d(\boldsymbol{N}) \rho) \\
& +\operatorname{Tr}\left(\sin ^{2}\left(\frac{\theta_{0}}{2} \sqrt{\boldsymbol{N}+1}\right)[d(\boldsymbol{N}+1)-d(\boldsymbol{N})] \rho\right) \\
& =V(\rho)+\operatorname{Tr}\left(\sin ^{2}\left(\frac{\theta_{0}}{2} \sqrt{\boldsymbol{N}+1}\right)[d(\boldsymbol{N}+1)-d(\boldsymbol{N})] \rho\right) .
\end{aligned}
$$

In particular,

$$
\begin{aligned}
& Q_{V}(\rho,+1)=-\operatorname{Tr}\left(\sin ^{2}\left(\frac{\theta_{0}}{2} \sqrt{\boldsymbol{N}+1}\right)[d(\boldsymbol{N}+1)-d(\boldsymbol{N})] \rho\right) \\
& =-\sum_{n \in \mathbb{N}} \rho_{n n}[2(n-\bar{n})+1] \sin ^{2}\left(\frac{\theta_{0}}{2} \sqrt{n+1}\right)
\end{aligned}
$$

Finally, take $u=-1$. Using (14) above and (12)(13) in Appendix $\mathbb{B}, \mathbb{E}\left[V\left(\rho_{k+1}\right) \mid \rho_{k}=\rho, u_{k}=-1\right]=$ $\operatorname{Tr}\left(\sin ^{2}\left(\frac{\theta_{0}}{2} \sqrt{\boldsymbol{N}}\right) d(\boldsymbol{N}-1) \rho\right)+\operatorname{Tr}\left(\cos ^{2}\left(\frac{\theta_{0}}{2} \sqrt{\boldsymbol{N}}\right) d(\boldsymbol{N}) \rho\right)$. By summing and subtracting $\operatorname{Tr}\left(\sin ^{2}\left(\frac{\theta_{0}}{2} \sqrt{\boldsymbol{N}}\right) d(\boldsymbol{N}) \rho\right)$,

$$
\begin{aligned}
& \mathbb{E}\left[V\left(\rho_{k+1}\right) \mid \rho_{k}=\rho, u_{k}=-1\right] \\
& =\operatorname{Tr}(d(\boldsymbol{N}) \rho)+\operatorname{Tr}\left(\sin ^{2}\left(\frac{\theta_{0}}{2} \sqrt{\boldsymbol{N}}\right)[d(\boldsymbol{N}-1)-d(\boldsymbol{N})] \rho\right) \\
& =V(\rho)+\operatorname{Tr}\left(\sin ^{2}\left(\frac{\theta_{0}}{2} \sqrt{\boldsymbol{N}}\right)[d(\boldsymbol{N}-1)-d(\boldsymbol{N})] \rho\right) .
\end{aligned}
$$

In particular,

$$
\begin{aligned}
Q_{V}(\rho,-1) & =-\operatorname{Tr}\left(\sin ^{2}\left(\frac{\theta_{0}}{2} \sqrt{\boldsymbol{N}}\right)[d(\boldsymbol{N}-1)-d(\boldsymbol{N})] \rho\right), \\
& =\sum_{n \in \mathbb{N}} \rho_{n n}[2(n-\bar{n})-1] \sin ^{2}\left(\frac{\theta_{0}}{2} \sqrt{n}\right) .
\end{aligned}
$$

\section{Proof of Lemma 1}

Assume that $N_{0}$ is even (otherwise one may take $N_{0}+1$ instead of $N_{0}$ in this proof). Define the function $\eta: \mathbb{N} \rightarrow \mathbb{R}$ by

$$
\eta(\ell)=\left[\frac{2}{\theta_{0}}\left(\ell \frac{\pi}{2}+\frac{\pi}{4}\right)\right]^{2} .
$$

By definition, one has $\frac{\theta_{0}}{2} \sqrt{\eta(\ell)}=\ell \frac{\pi}{2}+\frac{\pi}{4}$ for all $\ell \in \mathbb{N}$. Let $h=\pi / 4-\arcsin (\sqrt{1 / 2-a})$. Using the definition of $h$ and the symmetries of the function $\sin ^{2}(\cdot)$, it is easy to show that

$$
1 / 2-a \leq \sin ^{2}(x+\pi / 4) \leq a+1 / 2, \quad \forall x \in[-h, h] .
$$

Let $\bar{\ell} \in \mathbb{N}$ be even and big enough such that the following two conditions are simultaneously met:

$$
\eta(\bar{\ell})>N_{0} / 2+N, \quad \frac{1}{8} \theta_{0} N_{0} / \sqrt{\eta(\bar{\ell})-N_{0} / 2} \leq h .
$$

Now, take $\bar{N}=\lfloor\eta(\bar{\ell})\rceil-\frac{N_{0}}{2}+1>N$, where $\lfloor\eta\rceil$ denotes the greatest integer which is less or equal to $\eta$. By construction, $\eta(\bar{\ell})$ is in-between the points $\bar{N}+N_{0} / 2-1$ and $\bar{N}+N_{0} / 2$, and hence it is in the interval $\left[\bar{N}, \bar{N}+N_{0}-1\right]$. Then, for $n=$ $\bar{N}, \ldots, \bar{N}+N_{0}-1$, one has that $|n-\eta(\bar{\ell})|<N_{0} / 2$. Consider the function $\phi(x)=\frac{\theta_{0}}{2} \sqrt{x}$. From the fact that $\phi^{\prime}(x)=\frac{\theta_{0}}{4 \sqrt{x}}$, by the mean value theorem applied to the function $\phi$ and the second inequality in (20), one obtains

$\left|\frac{\theta_{0}}{2} \sqrt{n}-\frac{\theta_{0}}{2} \sqrt{\eta(\bar{\ell})}\right|<h, \quad$ for $n=\bar{N}, \ldots, \bar{N}+N_{0}-1$.

Then, the proof follows easily from (18), (19) and the fact that $\sin ^{2}(x-\ell \pi / 2)=\sin ^{2}(x)$, for every even $\ell \in \mathbb{N}$.

\section{E. Proof of Lemma 2}

Proof of the first claim: Let $u \in\{-1,0,1\}, \rho \in D_{*}$. Recall that $W(\rho)=-\sum_{n \in \mathbb{N}} \rho_{n n}^{2}$. Since $\operatorname{Tr}(\rho)=\sum_{n \in \mathbb{N}} \rho_{n n}=$ 1 , then $-1=-\sum_{n \in \mathbb{N}} \rho_{n n} \leq W(\rho) \leq 0$. Now, by $\left(10, \mathbb{E}\left[W\left(\rho_{k+1}\right) \mid \rho_{k}=\rho, u_{k}=u\right]=p_{g, k} W\left(\rho_{k+1}^{g}\right)+\right.$ $p_{e, k} W\left(\rho_{k+1}^{e}\right)$, where $p_{g, k}, p_{e, k} \geq 0$ with $p_{g, k}+p_{e, k}=1$. Thus $-1 \leq \mathbb{E}\left[W\left(\rho_{k+1}\right) \mid \rho_{k}=\rho, u_{k}=u\right] \leq 0$. Since $Q_{W}(\rho, u)$ is the difference of two numbers that are inbetween -1 and 0 , one concludes that $\left|Q_{W}(\rho, u)\right| \leq 1$.

The second, third and fourth claims, are immediate from (15), (16) and (17) in Appendix C] respectively.

\section{F. Proof of Lemma 3}

Proof of the first claim: Let $\rho \in D_{m_{0}}$. By (8)-(9) in Appendix B, $\boldsymbol{M}_{g}(0) \rho \boldsymbol{M}_{g}^{\dagger}(0)+\boldsymbol{M}_{e}(0) \rho \boldsymbol{M}_{e}^{\dagger}(0)=\rho$. Taking $\rho_{k}=\rho$ in $u_{k}=0$ in (1), define

$$
\rho^{y} \triangleq \rho_{k+1}^{y}=\frac{\boldsymbol{M}_{y}(0) \rho \boldsymbol{M}_{y}^{\dagger}(0)}{\operatorname{Tr}\left(\boldsymbol{M}_{y}(0) \rho \boldsymbol{M}_{y}^{\dagger}(0)\right)}, \quad \text { for } y=g, e .
$$

Hence, $\alpha \rho^{g}+(1-\alpha) \rho^{e}=\rho$, where $\alpha \triangleq p_{g, k}=$ $\operatorname{Tr}\left(\boldsymbol{M}_{g}(0) \rho \boldsymbol{M}_{g}^{\dagger}(0)\right)$. In particular, $\alpha \rho_{n n}^{g}+(1-\alpha) \rho_{n n}^{e}=$ $\rho_{n n}$, for $n \in \mathbb{N}$. Note that, if $\alpha=0$, then $\boldsymbol{M}_{g}(0) \rho \boldsymbol{M}_{g}^{\dagger}(0)=$ 0 , and so $\rho^{e}=\rho$. Similarly, $\alpha=1$ implies $\rho^{g}=\rho$. Thus, the identity $\alpha \rho_{n n}^{g}+(1-\alpha) \rho_{n n}^{e}=\rho_{n n}$, for $n \in \mathbb{N}$, still holds when $\alpha=0$ or $\alpha=1$. From (1), (7) and $\alpha=p_{g, k}$, one has

$$
\begin{aligned}
& Q_{W}(\rho, 0)=W(\rho)-\left[p_{g, k} W\left(\rho_{k+1}^{g}\right)+p_{e, k} W\left(\rho_{k+1}^{e}\right)\right] \\
& =\sum_{n \in \mathbb{N}} \alpha\left(\rho_{n n}^{g}\right)^{2}+(1-\alpha)\left(\rho_{n n}^{e}\right)^{2}-\left[\alpha \rho_{n n}^{g}+(1-\alpha) \rho_{n n}^{e}\right]^{2} \\
& =\alpha(1-\alpha) \sum_{n \in \mathbb{N}}\left[\rho_{n n}^{g}-\rho_{n n}^{e}\right]^{2} \geq 0,
\end{aligned}
$$

\footnotetext{
${ }^{6}$ More precisely, $\sin ^{2}(\pi / 2-x)=1-\cos ^{2}(\pi / 2-x)=1-\sin ^{2}(x)$
} 
thereby showing the first part of the first claim.

If $\rho=|m\rangle\langle m|$ for some $m \in \mathbb{N}$ with $0<\alpha<1$, then (8) - (9) in Appendix B imply that $\rho^{g}=\rho^{e}=\rho$, and so $Q_{W}(\rho, 0)=0$. Now, one shows that $\rho=|m\rangle\langle m|$ for some $m \in \mathbb{N}$ whenever $Q_{W}(\rho, 0)=0$. Suppose $Q_{W}(\rho, 0)=0$. Then, (21) implies that $\alpha=0$, or $\alpha=1$, or $\rho_{n n}^{g}=\rho_{n n}^{e}$ for all $n \in \mathbb{N}$ with $0<\alpha<1$. Assume that $\alpha=0$. Hence, $\boldsymbol{M}_{g}(0) \rho \boldsymbol{M}_{g}^{\dagger}(0)=\sum_{n \in \mathbb{N}} \rho_{n n} \cos ^{2}\left(\frac{\phi_{0} n+\phi_{R}}{2}\right)|n\rangle\langle n|=0$ by (8) in Appendix B. Suppose that $\rho \neq|m\rangle\langle m|$ for every $m \in \mathbb{N}$. Thus, there exists $n_{1}, n_{2} \in \mathbb{N}$ with $n_{1} \neq n_{2}$, $\rho_{n_{1}, n_{1}}>0, \rho_{n_{2}, n_{2}}>0$. Recall that $\sin \left(x_{1}\right)= \pm \sin \left(x_{2}\right)$ if and only if $x_{1}+x_{2}=\ell \pi$ or $x_{2}-x_{1}=\ell \pi$, where $\ell$ is an integer. Therefore, $\sin \left(\frac{\phi_{0} n_{1}+\phi_{R}}{2}\right)= \pm \sin \left(\frac{\phi_{0} n_{2}+\phi_{R}}{2}\right)$, which contradicts the assumptions that $\phi_{0} / \pi$ is an irrational number and $\phi_{R}=\pi / 2-\bar{n} \phi_{0}$. One has shown that $\rho=|m\rangle\langle m|$ for some $m \in \mathbb{N}$ whenever $\alpha=0$. If $\alpha=1$, or $\rho_{n n}^{g}=\rho_{n n}^{e}$ for all $n \in \mathbb{N}$ with $0<\alpha<1$, then from similar arguments and computations one also concludes that $\rho=|m\rangle\langle m|$ for some $m \in \mathbb{N}$.

Proof of the second claim: Let $m \in \mathbb{N}$ and take $\rho=$ $|m\rangle\langle m| \in D_{*}$. It is clear that $W(\rho)=-\sum_{n \in \mathbb{N}} \rho_{n n}^{2}=-1$. From 10]-13] in Appendix B] one has that:

$$
\begin{aligned}
& \left(\boldsymbol{M}_{g}(+1) \rho \boldsymbol{M}_{g}^{\dagger}(+1)\right)_{n n}=\delta(n, m+1) \sin ^{2}\left(\frac{\theta_{0}}{2} \sqrt{m+1}\right), \\
& \left(\boldsymbol{M}_{e}(+1) \rho \boldsymbol{M}_{e}^{\dagger}(+1)\right)_{n n}=\delta(n, m) \cos ^{2}\left(\frac{\theta_{0}}{2} \sqrt{m+1}\right), \\
& \left(\boldsymbol{M}_{g}(-1) \rho \boldsymbol{M}_{g}^{\dagger}(-1)\right)_{n n}=\delta(n, m) \cos ^{2}\left(\frac{\theta_{0}}{2} \sqrt{m}\right), \\
& \left(\boldsymbol{M}_{e}(-1) \rho \boldsymbol{M}_{e}^{\dagger}(-1)\right)_{n n}=\delta(n+1, m) \sin ^{2}\left(\frac{\theta_{0}}{2} \sqrt{m}\right),
\end{aligned}
$$

where $\delta(n, m)$ is the usual Kronecker delta: $\delta(n, m)=0$ if $n \neq m$, and $\delta(n, m)=1$ if $n=m$. In particular:

$$
\begin{aligned}
& \operatorname{Tr}\left(\boldsymbol{M}_{g}(+1) \rho \boldsymbol{M}_{g}^{\dagger}(+1)\right)=\sin ^{2}\left(\frac{\theta_{0}}{2} \sqrt{m+1}\right), \\
& \operatorname{Tr}\left(\boldsymbol{M}_{e}(+1) \rho \boldsymbol{M}_{e}^{\dagger}(+1)\right)=\cos ^{2}\left(\frac{\theta_{0}}{2} \sqrt{m+1}\right), \\
& \operatorname{Tr}\left(\boldsymbol{M}_{g}(-1) \rho \boldsymbol{M}_{g}^{\dagger}(-1)\right)=\cos ^{2}\left(\frac{\theta_{0}}{2} \sqrt{m}\right), \\
& \operatorname{Tr}\left(\boldsymbol{M}_{e}(-1) \rho \boldsymbol{M}_{e}^{\dagger}(-1)\right)=\sin ^{2}\left(\frac{\theta_{0}}{2} \sqrt{m}\right), \\
& \sum_{n \in \mathbb{N}}\left(\frac{\boldsymbol{M}_{y}(u) \rho \boldsymbol{M}_{y}^{\dagger}(u)}{\operatorname{Tr}\left(\boldsymbol{M}_{y}(u) \rho \boldsymbol{M}_{y}^{\dagger}(u)\right)}\right)_{n n}^{2}=1, \text { for } u= \pm 1, y=g, e
\end{aligned}
$$

(assuming no division by 0 ). Now, using (1) and the above computations, one gets

$$
\begin{aligned}
& \mathbb{E}\left[W\left(\rho_{k+1}\right) \mid \rho_{k}=\rho, u_{k}= \pm 1\right] \\
& =p_{g, k} W\left(\rho_{k+1}^{g}\right)+p_{e, k} W\left(\rho_{k+1}^{e}\right) \\
& =-\sum_{y=g, e}\left[\operatorname{Tr}\left(\boldsymbol{M}_{y}( \pm 1) \rho \boldsymbol{M}_{y}^{\dagger}( \pm 1)\right) \times\right. \\
& \left.\quad \times \sum_{n \in \mathbb{N}}\left(\frac{\boldsymbol{M}_{y}( \pm 1) \rho \boldsymbol{M}_{y}^{\dagger}( \pm 1)}{\operatorname{Tr}\left(\boldsymbol{M}_{y}( \pm 1) \rho \boldsymbol{M}_{y}^{\dagger}( \pm 1)\right)}\right)_{n n}^{2}\right] \\
& =-1=W(\rho) .
\end{aligned}
$$

Therefore, $Q_{W}(|m\rangle\langle m|, \pm 1)=0$.

\section{G. Proof of Proposition 2}

Fix $\rho \in \mathbb{D}_{*}$. Since $\operatorname{Tr}(d(\boldsymbol{N}) \rho)=\operatorname{Tr}(d(\boldsymbol{N}) \Delta \rho)$ and $\rho_{n n}=(\Delta \rho)_{n n}$ for $n \in \mathbb{N}$, the first two assertions are immediate from the definitions. As for the third and fourth assertions, let $|\psi\rangle=\sum_{m=0}^{\infty}\langle m \mid \psi\rangle|m\rangle \in \mathcal{H}$. Note that $\rho|m\rangle=\sum_{n=0}^{n_{\max }(\rho)} \rho_{m n}|n\rangle$, for $m \in \mathbb{N}$. Using (2)-(4):

$$
\begin{aligned}
& \boldsymbol{M}_{g}(0) \rho \boldsymbol{M}_{g}^{\dagger}(0)|\psi\rangle \\
& =\sum_{m, n=0}^{n_{\max }(\rho)} \rho_{m n} \cos \left(\frac{\phi_{0} m+\phi_{R}}{2}\right) \cos \left(\frac{\phi_{0} n+\phi_{R}}{2}\right)\langle m \mid \psi\rangle|n\rangle, \\
& \boldsymbol{M}_{e}(0) \rho \boldsymbol{M}_{e}^{\dagger}(0)|\psi\rangle \\
& =\sum_{m, n=0}^{n_{\max }(\rho)} \rho_{m n} \sin \left(\frac{\phi_{0} m+\phi_{R}}{2}\right) \sin \left(\frac{\phi_{0} n+\phi_{R}}{2}\right)\langle m \mid \psi\rangle|n\rangle, \\
& \boldsymbol{M}_{g}(+1) \rho \boldsymbol{M}_{g}^{\dagger}(+1)|\psi\rangle \\
& =\sum_{m=1, n=0}^{n_{\max }(\rho)+1} \rho_{m-1, n} \sin \left(\frac{\theta_{0}}{2} \sqrt{m}\right) \sin \left(\frac{\theta_{0}}{2} \sqrt{n+1}\right)\langle m \mid \psi\rangle|n+1\rangle, \\
& \boldsymbol{M}_{e}(+1) \rho \boldsymbol{M}_{e}^{\dagger}(+1)|\psi\rangle \\
& =\sum_{m, n=0}^{n_{\max }(\rho)} \rho_{m n} \cos \left(\frac{\theta_{0}}{2} \sqrt{m+1}\right) \cos \left(\frac{\theta_{0}}{2} \sqrt{n+1}\right)\langle m \mid \psi\rangle|n\rangle, \\
& \boldsymbol{M}_{g}(-1) \rho \boldsymbol{M}_{g}^{\dagger}(-1)|\psi\rangle \\
& =\sum_{m, n=0}^{n_{\max }(\rho)} \rho_{m n} \cos \left(\frac{\theta_{0}}{2} \sqrt{m}\right) \cos \left(\frac{\theta_{0}}{2} \sqrt{n}\right)\langle m \mid \psi\rangle|n\rangle, \\
& \boldsymbol{M}_{e}(-1) \rho \boldsymbol{M}_{e}^{\dagger}(-1)|\psi\rangle \\
& =\sum_{m=0, n=1}^{n_{\max }(\rho)} \rho_{m+1, n} \sin \left(\frac{\theta_{0}}{2} \sqrt{m+1}\right) \sin \left(\frac{\theta_{0}}{2} \sqrt{n}\right)\langle m \mid \psi\rangle|n-1\rangle \text {. }
\end{aligned}
$$

Since $\Delta \rho \in D_{*} \subset \mathbb{D}_{*}, n_{\max }(\Delta \rho)=n_{\max }(\rho)$ and $(\Delta \rho)_{n n}=\rho_{n n}$, the proof is straightforward from (8)- (13) in Appendix B

\section{REFERENCES}

[1] H. Amini, R.A. Somaraju, I. Dotsenko, C. Sayrin, M. Mirrahimi, and P. Rouchon. Feedback stabilization of discrete-time quantum systems subject to non-demolition measurements with imperfections and delays. Automatica, 49(9):2683-2692, September 2013.

[2] M. Brune, S. Haroche, J.-M. Raimond, L. Davidovich, and N. Zagury. Manipulation of photons in a cavity by dispersive atom-field coupling: Quantum-nondemolition measurements and generation of "Schrödinger cat" states. Physical Review A, 45(7):5193-5214, 1992.

[3] J. B. Conway. A Course in Operator Theory. American Mathematical Society, 2000.

[4] S. Haroche and J.M. Raimond. Exploring the Quantum: Atoms, Cavities and Photons. Oxford University Press, 2006.

[5] H.J. Kushner. Introduction to Stochastic Control. Holt, Rinehart and Wilson, INC., 1971.

[6] M.A. Nielsen and I.L. Chuang. Quantum Computation and Quantum Information. Cambridge University Press, 2000.

[7] M. Reed and B. Simon. Methods of Modern Mathematical Physics. Functional Analysis (Vol. 1). Academic Press, 1980.

[8] X. Zhou, I. Dotsenko, B. Peaudecerf, T. Rybarczyk, C. Sayrin, J.M. Raimond S. Gleyzes, M. Brune, and S. Haroche. Field locked to Fock state by quantum feedback with single photon corrections. Physical Review Letter, 108:243602, 2012. 